\title{
D-branes in a topologically nontrivial $B$-field
}

\author{
Anton Kapustin \\ School of Natural Sciences, Institute for Advanced Study \\ Olden Lane, Princeton, NJ 08540 \\ kapustin@ias.edu
}

\begin{abstract}
We study global worldsheet anomalies for open strings ending on several coincident $\mathrm{D}$-branes in the presence of a $B$-field. We show that cancellation of anomalies is made possible by a correlation between the t'Hooft magnetic flux on the D-branes and the topological class of the $B$-field. One application of our results is a proper understanding of the geometric nature of the gauge field living on D-branes: rather than being a connection on a vector bundle, it is a connection on a module over a certain noncommutative algebra. Our argument works for a general closed string background. We also explain why in the presence of a topologically nontrivial $B$-field whose curvature is pure torsion D-branes represent classes in a twisted $\mathrm{K}$-theory, as conjectured by E. Witten.
\end{abstract}

e-print archive: http://xxx.lanl.gov/hep-th/9909089 


\section{Introduction and summary}

The goal of this paper is to understand the conditions for cancellation of global worldsheet anomalies for open strings ending on D-branes. In the case when all $\mathrm{D}$-branes are noncoincident, this has been achieved in [1]. It turns out that interesting new phenomena arise when one considers coincident $\mathrm{D}$-branes with nonabelian gauge fields. In particular, noncommutative geometry makes an appearance. Recently, it has been argued by many authors that the gauge field on D-branes is better understood as a connection on a module over some noncommutative algebra rather than as a connection on a vector bundle. This has been demonstrated for D-branes in flat space in the presence of a constant $B$-field [2]. Our setup is much more general (arbitrary metric and $B$ field), so it is nice to see that the same interpretation of the gauge field arises again. On the other hand, we will be concerned only with the topological properties of the gauge field, leaving its dynamics aside.

\subsection{Review of twisted K-theory}

The main motivation for this study was the interpretation of D-brane charges in terms of K-theory [3, 4], so we begin by reviewing it. It has been suspected for some time that D-brane charges are most naturally understood as classes in K-theory rather than as integer cohomology classes [3]. The basic reason for this is that D-branes carry vector bundles. In Ref. [4] E. Witten showed that the relation between Dbranes and K-theory can be understood very simply using some ideas of A. Sen [5]. For IIB string theory one considers a configuration of equal number of D9 and anti-D9-branes carrying vector bundles $E$ and $F$. The pair $(E, F)$ defines a class in K-theory. It is easy to see that creation of virtual brane-antibrane pairs from vacuum does not change the $\mathrm{K}$ theory class. Thus the stable state into which the brane-antibrane system settles after tachyons condense can be labeled by this class. It is also very plausible that if pairs $(E, F)$ and $\left(E^{\prime}, F^{\prime}\right)$ have the same $\mathrm{K}$-theory class, then they lead to the same stable state, as one can be converted to the other by a virtual process. Conversely, Witten explained how, given a D-brane wrapped on a submanifold, one can construct a K-theory class it represents. 
In the presence of the Neveu-Schwarz B-field these arguments should be modified. One way to see this is to note $[4,1]$ that a single D-brane can be wrapped on a submanifold $Q$ if and only if the normal bundle $N$ of $Q$ satisfies:

$$
W_{3}(N)=[H]_{Q}
$$

Here $W_{3}(N) \in H^{3}(Q, \mathbb{Z})$ is the image of the second Stiefel-Whitney class $w_{2}(N)$ under the Bockstein homomorphism, $[H]$ is the integer cohomology class whose de Rham representative is $H=d B$, and $[H]_{Q}$ is its restriction to $Q$. Eq. (1) is the condition of cancellation of global anomalies for open strings ending on $Q$ [1]. It refines the usual statement that the restriction of $H$ to the worldvolume of a D-brane must be trivial in de Rham cohomology. When $[H]_{Q}=0$ the condition (1) says that $N$ has $\operatorname{Spin}^{\text {c }}$ structure, and enables one to construct a class in the $\mathrm{K}$-theory of space-time $X$ corresponding to a D-brane wrapped on $Q$ [4]. When $[H]_{Q}$ is nonzero, the submanifold $Q$ does not represent a K-theory class.

In Ref. [4] it has been proposed that when $[H]$ belongs to a torsion subgroup of $H^{3}(M, \mathbb{Z})$, D-brane charges take values in a certain "twisted" version of K-theory $K_{H}(M)$. (In mathematical literature it is sometimes called K-theory with local coefficients.) To motivate the definition of $K_{H}(M)$, let us recall that the space of global sections $\Gamma(M, E)$ of a (finite-dimensional) vector bundle $E$ is a (finitely generated) projective module over the algebra of continuous $\mathbb{C}$-valued functions on $M$ Cont $_{M}(\mathbb{C})$. (A projective module is a module which is a direct summand of a free module. $\Gamma(M, E)$ is projective for any $E$ because any $E$ is a direct summand of a trivial vector bundle.) Since $M$ is completely determined by the algebra $\operatorname{Cont}_{M}(\mathbb{C})$, it should be possible to define $K(M)$ directly in terms of $\operatorname{Cont}_{M}(\mathbb{C})$. Such a definition is indeed possible: for any algebra $\mathrm{A}$ one can define its $\mathrm{K}$-group $K(\mathrm{~A})$ as the Grothendieck group of finitely generated projective modules over A. By a theorem of Serre-Swan $[6,7]$ the category of finitely generated projective modules over $\operatorname{Cont}_{M}(\mathbb{C})$ is equivalent to the category of finitedimensional vector bundles over $M$, hence $K\left(\right.$ Cont $\left._{M}(\mathbb{C})\right)=K(M)$.

Now it is plausible that when the B-field is present, we must replace Cont $_{M}(\mathbb{C})$ with some other (noncommutative) algebra $A$ and consider $K(\mathrm{~A})$. Morally speaking, this follows from the lore that when the Bfield is switched on, $M$ becomes a noncommutative space in the sense of Connes [8, 2]. 
A natural class of algebras is provided by so-called Azumaya algebras [9]. An Azumaya algebra of rank $m$ over $M$ is a locally trivial algebra bundle over $M$ whose fiber is isomorphic to the algebra of $m \times m$ matrices $\operatorname{Mat}_{m}(\mathbb{C})$. A trivial example of an Azumaya algebra over $M$ is $\operatorname{Mat}_{m}(\mathbb{C}) \otimes \operatorname{Cont}_{M}(\mathbb{C})$, i.e. the algebra of $\operatorname{Mat}_{m}(\mathbb{C})$-valued functions on $M$. A slightly less trivial example is the algebra $\operatorname{End}(E)$ of all endomorphisms of a vector bundle $E$ over $M$. Two Azumaya algebras A and $\mathrm{A}^{\prime}$ over $M$ are said to be equivalent if there exist two vector bundles $E$ and $E^{\prime}$ over $M$ such that $\mathrm{A} \otimes \operatorname{End}(E)$ is isomorphic to $\mathrm{A}^{\prime} \otimes \operatorname{End}\left(E^{\prime}\right)$. In particular, an Azumaya algebra of the form $\operatorname{End}(E)$ is equivalent in this sense to $\operatorname{Cont}_{M}(\mathbb{C})$ for any $E$. This definition of equivalence is useful because the K-group of an Azumaya algebra depends only on its equivalence class [10]. Moreover, by a theorem of Serre [9] equivalence classes of Azumaya algebras over $M$ are classified by the torsion subgroup of $H^{3}(M, \mathbb{Z}) .{ }^{1}$ We will denote by $\delta(\mathrm{A})$ the class in $H^{3}(M, \mathbb{Z})$ corresponding to A. To summarize, for any torsion class $[H] \in H^{3}(M, \mathbb{Z})$ there is a unique equivalence class of Azumaya algebras and the corresponding $\mathrm{K}$-group $K_{H}(M)$. It is natural to conjecture [4] that $K_{H}(M)$ classifies D-brane charges when $[H]$ is a torsion class.

It possible to give a more down-to-earth description of Azumaya algebras and their modules. Let us pick an open cover $\left\{\mathcal{U}_{i}\right\}$ such that all $\mathcal{U}_{i}$ and their multiple overlaps are contractible. An element of an Azumaya algebra A of rank $m$ is a section of a vector bundle with fiber $\operatorname{Mat}_{m}(\mathbb{C})$, i.e. it is a collection of functions $R_{i}: \mathcal{U}_{i} \rightarrow \operatorname{Mat}_{m}(\mathbb{C})$ such that on double overlaps $\mathcal{U}_{i} \cap \mathcal{U}_{j}=\mathcal{U}_{i j}$ we have

$$
R_{i}=G_{i j} R_{j} G_{i j}^{-1}
$$

Here $G_{i j}$ are $G L(m, \mathbb{C})$-valued functions over $\mathcal{U}_{i j}$. Consistency of (2) on triple overlaps $\mathcal{U}_{i j k}$ requires

$$
G_{i j} G_{j k} G_{k i}=\zeta_{i j k}
$$

where $\zeta_{i j k}$ are $\mathbb{C}^{*}$-valued functions. It is easy to see that $\zeta_{i j k}$ define a Čech cocycle, and therefore an element $\zeta \in H^{2}\left(M, \operatorname{Cont}_{M}\left(\mathbb{C}^{*}\right)\right)$. Here $\mathbb{C}^{*}$ is the group of nonzero complex numbers. $\zeta$ is a measure of nontriviality of the Azumaya algebra A. By a well-known isomorphism

\footnotetext{
${ }^{1}$ This subgroup is called the topological Brauer group of $M$ and is denoted $\operatorname{Br}(M)$.
} 
$H^{2}\left(M, \operatorname{Cont}_{M}\left(\mathbb{C}^{*}\right)\right) \simeq H^{3}(M, \mathbb{Z})$ we can reinterpret $\zeta$ as a class in $H^{3}(M, \mathbb{Z})$; this is the class $\delta(\mathrm{A})$ mentioned above.

A module over an Azumaya algebra can be described as follows. Suppose we are given a set of functions $h_{i j}: \mathcal{U}_{i} \rightarrow G L(n, \mathbb{C})$ satisfying on triple overlaps

$$
h_{i j} h_{j k} h_{k i}=\zeta_{i j k}
$$

where the cocycle $\zeta$ is the same as in (3). Consider a vector bundle with fiber $\mathbb{C}^{n m}$ and transition functions

$$
h_{i j}^{-1 T} \otimes G_{i j}
$$

First of all, such a bundle makes sense since the transition functions satisfy the usual "untwisted" gluing condition on triple overlaps. Second, its fiber

$$
\mathbb{C}^{n m} \simeq \mathbb{C}^{m} \oplus \cdots \oplus \mathbb{C}^{m}
$$

is naturally a module over $\operatorname{Mat}_{m}(\mathbb{C})$, the fiber of $A$. Third, it is easy to check that the obvious "fiberwise" action of A makes it into a module over A. It can be shown that this module is projective if $M$ is a nice enough space (compact, for example). Moreover, all projective modules of $A$ arise in this way. ${ }^{2}$

\subsection{Azumaya algebras and D-branes}

Now we are going to explain the relation between global worldsheet anomalies and Azumaya modules. The basic idea is rather simple. The starting point for the Sen-Witten construction of stable D-branes in IIB string theory is a configuration containing $n$ D9-branes and $n$ antiD9-branes. When $[H]=0$, the D-branes carry a principal bundle with

\footnotetext{
${ }^{2}$ Let us give a sketch of a proof. First, a projective module over $\mathrm{A}$ is also a projective module over $\operatorname{Cont}_{M}(\mathbb{C})$ and therefore corresponds to a vector bundle $E$ over $M$. Second, it can be shown that the fiber of $E$ must be a module over $\operatorname{Mat}_{m}(\mathbb{C})$. Third, any module over $\operatorname{Mat}_{m}(\mathbb{C})$ has the form (6) for some $n$ [11]. Fourth, the action of A on any given fiber $E_{x}$ of $E$ generates a subalgebra $A_{x}$ of $\operatorname{End}\left(E_{x}\right) \simeq \operatorname{Mat}_{n m}(\mathbb{C})$ isomorphic to $\operatorname{Mat}_{m}(\mathbb{C})$. Fifth, let the transition functions for $E$ be $g_{i j}$. It is easy to see that in order for the fiberwise action of $A$ to be consistent on double overlaps, $G_{i j}^{-1} g_{i j}$ must be in the centralizer $C\left(\mathrm{~A}_{x}\right)$ of $\mathrm{A}_{x}$. Since both $\mathrm{A}_{x}$ and $\operatorname{End}\left(E_{x}\right)$ are central simple algebras, by Corollary 3.16 of [11] $\operatorname{End}\left(E_{x}\right) \simeq \mathrm{A}_{x} \otimes C\left(\mathrm{~A}_{x}\right)$, and it follows that there exists a trivialization of $E$ such that $g_{i j}$ have the form (5).
} 
structure group $U(n)$. We will show that when $[H]$ is nonzero, the D-branes carry a principal $S U(n) / \mathbb{Z}_{n}$ bundle which cannot be lifted to a $U(n)$ bundle. The obstruction to lifting an $S U(n) / \mathbb{Z}_{n}$ bundle to a $U(n)$ bundle is a certain class in $H^{3}(M, \mathbb{Z})$ closely related to the t'Hooft magnetic flux. We will prove that cancellation of global worldsheet anomalies requires that this class be equal to $[H]$.

An $S U(n) / \mathbb{Z}_{n}$ bundle without $U(n)$ structure can described in terms of transition functions as follows. Take an associated vector bundle in the adjoint representation of $S U(n) / \mathbb{Z}_{n}$. Its section is given by a collection of functions $f_{i}: \mathcal{U}_{i} \rightarrow u(n)$, where $u(n)$ is the Lie algebra of $S U(n) / \mathbb{Z}_{n}$, i.e. the space of all hermitian $n \times n$ matrices. On double overlaps they satisfy

$$
f_{i}=h_{i j} f_{j} h_{i j}^{-1},
$$

where $h_{i j}$ are $U(n)$-valued matrices. Consistency on triple overlaps requires

$$
h_{i j} h_{j k} h_{k i}=\zeta_{i j k},
$$

where $\zeta_{i j k}$ are $U(1)$-valued functions. The cohomology class of $\zeta$ in $H^{2}\left(M\right.$, Cont $\left._{M}(U(1))\right) \simeq H^{3}(M, \mathbb{Z})$ is the obstruction to lifting the $S U(n) / \mathbb{Z}_{n}$ structure to a $U(n)$ structure.

Comparing (8) and (4) we discover that an $S U(n) / \mathbb{Z}_{n}$ bundle without $U(n)$ structure corresponds naturally to a module over an Azumaya algebra $A$ with $\delta(A)=\zeta$. Since we have both D9-branes and anti-D9branes, we get a pair of modules over the same algebra $A$ which define a class in $K_{H}(M)$. It is also easy to see that creation and annihilation of branes does not change this class.

Furthermore, the gauge field in the fundamental representation of $U(n)$ (whose holonomy enters the open string path integral, see below) cannot be a connection on a rank $n$ vector bundle over $M$, since there is no such bundle around. Instead, the gauge field is a connection on the Azumaya module defined above.

Thus cancellation of global worldsheet anomalies for open strings ending on D9-branes is the reason why D-brane charges take values in $K_{H}(M)$. More generally, one can analyze these anomalies for open strings ending on D-branes of arbitrary dimension. This yields consistency conditions for wrapping multiple D-branes on a submanifold $Q \subset M$. To formulate these consistency conditions we need to be more 
precise about the relation between the t'Hooft magnetic flux and the obstruction to having $U(n)$ structure. The t'Hooft magnetic flux is a cohomology class $y \in H^{2}\left(Q, \mathbb{Z}_{n}\right)$ which measures the obstruction to lifting an $S U(n) / \mathbb{Z}_{n}$ structure to an $S U(n)$ structure. Consider a short exact sequence of groups

$$
0 \rightarrow \mathbb{Z} \rightarrow \mathbb{Z} \rightarrow \mathbb{Z}_{n} \rightarrow 0
$$

where the second arrow is multiplication by $n$ and the third arrow is reduction modulo $n$. It leads to a long exact sequence in cohomology

$$
\ldots H^{2}\left(Q, \mathbb{Z}_{n}\right) \rightarrow H^{3}(Q, \mathbb{Z}) \rightarrow H^{3}(Q, \mathbb{Z}) \ldots
$$

The homomorphism $H^{2}\left(Q, \mathbb{Z}_{n}\right) \rightarrow H^{3}(Q, \mathbb{Z})$ is a Bockstein homomorphism and will be denoted $\beta^{\prime}$. As explained in more detail below, the obstruction to having a $U(n)$ structure is precisely $\beta^{\prime}(y)$. We will show that for bosonic strings cancellation of global anomalies requires

$$
\beta^{\prime}(y)=[H]_{Q} .
$$

For superstrings we find that global anomalies cancel if

$$
\beta^{\prime}(y)+W_{3}(N)=[H]_{Q} .
$$

This generalizes (1). We will also explain the analogue of these conditions for Type I D-branes.

\subsection{The mechanism of anomaly cancellation}

Showing that global worldsheet anomalies cancel if (11) or (12) are satisfied is unfortunately rather tedious. (For an analogous argument in the abelian case see [1], Section 6. The argument for Type I D9branes was sketched in [12].) This is because we tried to keep the discussion elementary yet reasonably rigorous. Let us briefly explain what is involved in the argument. For simplicity, consider the path integral for an open bosonic string in the presence of D9-branes:

$$
\int \mathcal{D} \xi \exp \left[\sqrt{-1} S_{N G}[\xi]+\sqrt{-1} \int_{\Sigma} \xi^{*} B\right] \operatorname{Tr} \operatorname{Hol}_{\partial \Sigma}(A) .
$$

Here $\xi$ is the map from the worldsheet $\Sigma$ to the target space $M, S_{N G}[\xi]$ is the Nambu-Goto action, $A$ is the $U(n)$ gauge field on the D9-branes, 
$\operatorname{Hol}_{\gamma}(A)$ is the holonomy of $A$ along the loop $\gamma$, and $\operatorname{Tr}$ is the trace taken in the fundamental representation of $U(n)$. It will be sufficient to consider the case when $\Sigma$ is a disc. The Nambu-Goto action is a welldefined function on the space of maps $\operatorname{Map}(\Sigma, M)$ from $\Sigma$ to $M$, but the other two factors are more problematic. Indeed, while $H=d B$ is a welldefined 3-form, $B$ itself may have "Dirac string singularities." Similarly, since the $S U(n) / \mathbb{Z}_{n}$ bundle cannot be lifted to a $U(n)$ bundle, the trace of holonomy of the gauge connection in the fundamental representation of $U(n)$ is not well-defined. This can be traced back to the "twisted" gluing condition (8).

In fact, both of these factors make perfect sense if we interpret them as sections of certain line bundles over $\operatorname{Map}(\Sigma, M)$ rather than as mere functions. Recall that the trace of holonomy of a connection on an ordinary vector bundle is a function on a free loop space $L M$ of $M$. We can define the trace of holonomy for our "connection" as well, but it turns out that it takes values in a certain nontrivial line bundle over $L M$. Similarly, the phase factor coming from the $B$-field is a section of a well-defined line bundle over $\operatorname{Map}(\Sigma, M)$.

We are actually interested not in holonomy around arbitrary loops $S^{1} \rightarrow M$, but around those loops which extend to a map $\Sigma \rightarrow M$ where $\Sigma$ is a disc. Thus we are interested not in the above-mentioned line bundle over $L M$ but in its pull-back to $\operatorname{Map}(\Sigma, M)$. We will show that the pull-back is trivial, and the phase factor coming from the Bfield provides its trivialization. As a result, although neither the trace of holonomy nor the phase coming from the B-field are functions on $\operatorname{Map}(\Sigma, M)$, their product is a well-defined function. This implies that the open string path integral is well-defined.

\subsection{Outline of the paper}

The paper is organized as follows. In Section II we recall how to define $B$-field in a topologically nontrivial situation. In Section III we describe the topology of the gauge fields living on D-branes. In Section IV we explain how one is supposed to understand the trace of holonomy of a connection in the fundamental representation of $U(n)$ in the presence of t'Hooft magnetic flux. In Section $V$ we define the phase factor $\exp \left(-\sqrt{-1} \int \xi^{*} B\right)$ following $[13,14]$ and show that the open string 
path integral for bosonic strings is well-defined if the condition (11) is satisfied. In Section VI we extend the discussion to superstrings and show that anomalies cancel if (12) is satisfied. As explained above, this implies that stable D-branes in Type IIB string theory are classified by $K_{H}(X)$. In Section VII we discuss what happens when $[H]$ is not a torsion class.

A word about conventions. We denote by $\mathbb{R}$ the field of real numbers, and by $Z$ the abelian subgroup of $\mathbb{R}$ generated by $2 \pi$. Of course, $Z$ is canonically isomorphic to the group of integers $\mathbb{Z}$. It is also a module over the ring $\mathbb{Z}$. We will normalize $H=d B$ so that its periods are integer multiples of $2 \pi$. Thus $[H]$ will be a class in $H^{3}(M, \mathrm{Z})$ rather than in $H^{3}(M, \mathbb{Z})$.

\section{The definition of the B-field}

The precise definition of the B-field is most conveniently formulated in the language of Čech-de Rham cohomology $[13,1]$. Choose an open cover $\mathcal{U}_{i}, i \in I$, of $M$, such that all $\mathcal{U}_{i}$, the double overlaps $\mathcal{U}_{i j}=\mathcal{U}_{i} \cap \mathcal{U}_{j}$, the triple overlaps $\mathcal{U}_{i j k}$, etc., are contractible (such a cover exists for any manifold.) On each $\mathcal{U}_{i}$ there is a well-defined real 2-form $B_{i}$. On double overlaps $\mathcal{U}_{i j}$ we have the relation

$$
B_{j}-B_{i}=d \Lambda_{i j}
$$

where $\Lambda_{i j}$ are real 1 -forms. These 1 -forms satisfy a consistency condition on triple overlaps $\mathcal{U}_{i j k}$ :

$$
\Lambda_{i j}+\Lambda_{j k}+\Lambda_{k i}=-\sqrt{-1} d \log \alpha_{i j k}
$$

where $\alpha_{i j k}$ are $U(1)$-valued 0 -forms. In turn, $\alpha_{i j k}$ satisfy a further constraint on quadruple overlaps $\mathcal{U}_{i j k l}$ :

$$
\alpha_{i j k} \alpha_{j k l}^{-1} \alpha_{i k l} \alpha_{i j l}^{-1}=1 \text {. }
$$

Equations (14-16) mean that $B$-field is a connection on a gerbe [15], as defined in [16]. However, we will not use this terminology here.

Equation (16) implies

$$
-\log \alpha_{i j k}+\log \alpha_{j k l}-\log \alpha_{i k l}+\log \alpha_{i j l}=\sqrt{-1} m_{i j k l},
$$


where $m_{i j k l} \in$ Z. $m_{i j k l}$ form a Čech cochain with values in Z. Moreover, it is easy to see from (17) that $m$ is a Cech cocycle and therefore represents a class in $H^{3}(M, \mathrm{Z})$. This class will be called $[H]$, because its de Rham representative is $H=d B$.

Note that the condition (16) means that the functions $\alpha_{i j k}$ form a 2-cocycle with values in $\operatorname{Cont}_{M}(U(1))$, the sheaf of $U(1)$-valued continuous functions on $M$. One can construct the corresponding class in $H^{2}\left(M\right.$, Cont $_{M}(U(1))$ directly from $[H]$ by the following standard argument. Consider an exponential exact sequence of sheaves

$$
0 \rightarrow \mathrm{Z} \rightarrow \mathrm{Cont}_{M}(\mathbb{R}) \rightarrow \mathrm{Cont}_{M}(U(1)) \rightarrow 0 .
$$

It induces a long exact sequence in cohomology which reads in part

$$
\begin{aligned}
\ldots H^{2}\left(M, \operatorname{Cont}_{M}(\mathbb{R})\right) & \rightarrow H^{2}\left(M, \operatorname{Cont}_{M}(U(1))\right) \\
& \rightarrow H^{3}(M, \mathrm{Z}) \rightarrow H^{3}\left(M, \operatorname{Cont}_{M}(\mathbb{R})\right) \ldots
\end{aligned}
$$

But the sheaf $\operatorname{Cont}_{M}(\mathbb{R})$ is fine, so $H^{p}\left(M, \operatorname{Cont}_{M}(\mathbb{R})\right)=0$ for all $p>0$. It follows that $H^{2}\left(M, \operatorname{Cont}_{M}(U(1))\right)$ is isomorphic to $H^{3}(M, \mathrm{Z})$. The cohomology class of $\alpha$ is mapped to $[H]$ under this isomorphism.

We are interested in the situation when we are given a submanifold $Q \subset M$ and the restriction of $H$ to $Q$ is trivial in de Rham cohomology, i.e. $[H]_{Q}$ is pure torsion. Until Section 5 we are going to work exclusively with objects on $Q$, so in order not to clutter notation we will omit the subscript $Q$ until further notice. Hopefully this will not cause confusion.

An open cover $\left\{\mathcal{U}_{i}\right\}$ of $M$ induces an open cover of $Q$ indexed by the same set $I$. By abuse of notation, we will continue to refer to the elements of the cover as $\mathcal{U}_{i}$. If $H$ is trivial in de Rham cohomology, there exists a 2-form $\tilde{B}$ such that $H=d B$. Then $B_{i}=\tilde{B}+\mu_{i}$, where $\mu_{i}$ is a real 1 -form on $\mathcal{U}_{i}$. From (14) it follows that on double overlaps we have

$$
\mu_{j}-\mu_{i}-\Lambda_{i j}=d \rho_{i j}
$$

for some real 0 -forms $\rho_{i j}$. From (15) and (20) it follows that on triple overlaps we have

$$
-\sqrt{-1}\left(\rho_{i j}+\rho_{j k}+\rho_{k i}\right)=\log \alpha_{i j k}-\log \zeta_{i j k},
$$

where $\zeta_{i j k}$ are complex numbers with unit modulus. The numbers $\zeta_{i j k}$ form a Cech cochain with values in $U(1)$. In fact (17) implies that $\zeta$ 
is a Čech cocycle and so defines a class in $H^{2}(Q, U(1))$. The origin of this class can be understood more abstractly as follows. Consider an exponential exact sequence of groups

$$
0 \rightarrow Z \rightarrow \mathbb{R} \rightarrow U(1) \rightarrow 0 .
$$

It leads to a long exact sequence in the cohomology of $Q$ which reads in part

$$
\ldots H^{2}(Q, U(1)) \rightarrow H^{3}(Q, \mathrm{Z}) \rightarrow H^{3}(Q, \mathbb{R}) \ldots
$$

The first arrow here is a Bockstein homomorphism which we will call $\beta$. Since the cohomology class of $H$ in $H^{3}(Q, \mathbb{R})$ is trivial, exactness implies that there is an element $\zeta \in H^{2}(Q, U(1))$ such that $\beta(\zeta)=[H]$. $\zeta$ is precisely the class we have constructed above using Cech cocycles.

\section{The topology of the gauge bundle on D-branes.}

It is usually said that $A$ (the gauge field on D-branes) is a connection on a $U(n)$ vector bundle over $Q$, but transforms nontrivially under the gauge transformation $B \rightarrow B+d \Lambda$, to wit $A \rightarrow A-\Lambda$. We need a more precise statement which would tell us how 1-forms $A_{i}$ on various patches are glued together. We regard $A$ as a collection of 1 -forms $\tilde{A}_{i}, i \in I$. We postulate that on double overlaps they satisfy

$$
\tilde{A}_{i}=g_{i j} \tilde{A}_{j} g_{i j}^{-1}+\sqrt{-1} g_{i j} d g_{i j}^{-1}-\Lambda_{i j} \text {. }
$$

Here $\Lambda_{i j}$ are the same 1-forms as in (14), and $g_{i j}$ are $U(n)$-valued functions. The transformation law (24) is not the correct transformation law for a connection on a vector bundle. It is not even immediately clear how to define the holonomy of such a "connection" $A$ around a loop. A natural definition exists only when the restriction of $H$ to $Q$ is trivial in de Rham cohomology. It will turn out that the trace of holonomy of $A$ takes values in a line bundle over the loop space $L Q$ rather than in complex numbers.

Apart from the last term in (24), the transformation law for $\tilde{A}_{i}$ is that of a connection on a vector bundle. To be precise, for a vector bundle the gluing functions $g_{i j}$ must satisfy

$$
g_{i j} g_{j k} g_{k i}=1 \text {. }
$$


We will impose instead

$$
g_{i j} g_{j k} g_{k i}=\alpha_{i j k}
$$

where $\alpha_{i j k}$ were defined in (15). The reason for this peculiar definition is that it will make the open string path integral well defined.

In order to understand in what sense $A$ is a "connection", we need to get rid of the last term in (24). Recall that when $B$ is flat, $\Lambda_{i j}$ satisfy (20). We define new 1 -forms $A_{i}$ by

$$
A_{i}=\tilde{A}_{i}-\mu_{i}
$$

Their transformation law follows from (24) and (20):

$$
A_{i}=h_{i j} A_{j} h_{i j}^{-1}+\sqrt{-1} h_{i j} d h_{i j}^{-1},
$$

where

$$
h_{i j}=g_{i j} e^{\sqrt{-1} \rho_{i j}} .
$$

The transformation law for $A_{i}$ looks like that of an ordinary connection on a vector bundle. However, the gluing functions $h_{i j}$ do not satisfy the usual condition (25) on triple overlaps. Instead one can show, using (26), (21), and (17) that they satisfy

$$
h_{i j} h_{i j} h_{k i}=\zeta_{i j k}
$$

Thus $h_{i j}$ do not define a $U(n)$ vector bundle over $Q$. Instead, as explained in Section I, they define a module $\Gamma$ over an Azumaya algebra A with $\delta(\mathrm{A})=\beta(\zeta)=[H]$.

Although the transition functions $h_{i j}$ taken in the fundamental representation of $U(n)$ do not define a vector bundle, they do define a vector bundle when taken in the adjoint representation of $U(n)$. This is because $h_{i j}$ fail to glue properly only modulo the elements of the center of $U(n)$, which is immaterial once one passed to the adjoint representation. Thus we obtain a rank $n^{2}$ bundle over $Q$ which we call Adj. It is easy to see that the 1 -forms $A_{i}$ lead to a well-defined connection on Adj. This enables one to define the holonomy of $A$ in the adjoint representation in the usual manner.

The bundle Adj has $U(n) / U(1)=S U(n) / \mathbb{Z}_{n}$ as its structure group. One can define a class $y$ in $H^{2}\left(Q, \mathbb{Z}_{n}\right)$ which measures the obstruction 
to lifting it to an $S U(n)$ bundle. This class is called the t'Hooft magnetic flux in the physics literature. Let us construct a Čech cocycle representing $y$. Set

$$
q_{i j}=\left(\operatorname{det} h_{i j}\right)^{1 / n}
$$

and define $S U(n)$-valued transition functions

$$
\tilde{h}_{i j}=q_{i j}^{-1} h_{i j}
$$

In general, they do not glue properly over triple overlaps; instead we have

$$
\tilde{h}_{i j} \tilde{h}_{j k} \tilde{h}_{k i}=y_{i j k} \text {. }
$$

Taking the determinant of both sides of (32) we see that $y_{i j k}$ is a 2cochain with values in $\mathbb{Z}_{n}$. Furthermore, it follows directly from the definition of $y$ that it is a 2-cocycle. This cocycle is the Cech representative of the t'Hooft magnetic flux.

For our purposes it is more useful to know if Adj can be lifted to a $U(n)$ bundle. This may be possible even if $y \neq 0$. Indeed, suppose $y$ is nonzero, but is a reduction modulo $n$ of an integer cohomology class $z$. This means that there exists a Z-valued Čech cocycle $z_{i j k}$ such that

$$
y_{i j k}=\exp \left(\frac{\sqrt{-1} z_{i j k}}{n}\right)
$$

A standard argument shows there exists a line bundle $\mathcal{L}$ over $Q$ whose first Chern class is $z .^{3}$ Let its transition functions be $t_{i j}$, i.e.

$$
\log t_{i j}+\log t_{j k}+\log t_{k i}=-\sqrt{-1} z_{i j k} .
$$

Then the $U(n)$-valued functions $u_{i j}=\tilde{h}_{i j} t_{i j}^{1 / n}$ satisfy

$$
u_{i j} u_{j k} u_{k i}=1 \text {. }
$$

This means that $u_{i j}$ define a vector bundle with structure group $U(n)$. It follows that a sufficient condition for being able to lift Adj to a $U(n)$ bundle is

$$
\exists z \in H^{2}(Q, Z) \quad y=z \bmod 2 \pi n .
$$

\footnotetext{
${ }^{3}$ First one uses the exact sequence $(19)$ and the fact that $\operatorname{Cont}_{Q}(\mathbb{R})$ is a fine sheaf to show that $H^{1}\left(Q, \operatorname{Cont}_{Q}(U(1))\right) \simeq H^{2}(Q, \mathrm{Z})$. Let $t$ be the preimage of $z \in H^{2}(Q, Z)$ under this isomorphism. The Cech cocycle $t_{i j}$ representing $t$ yields the transition functions for $\mathcal{L}$.
} 
It is easy to see that this condition is also necessary.

There is a convenient way to rewrite this criterion. Consider a short exact sequence of groups

$$
0 \rightarrow \mathrm{Z} \rightarrow \mathrm{Z} \rightarrow \mathbb{Z}_{n} \rightarrow 0
$$

where the second arrow is multiplication by $n$. This exact sequence leads to a long exact sequence in cohomology

$$
\ldots H^{2}(Q, \mathrm{Z}) \rightarrow H^{2}\left(Q, \mathbb{Z}_{n}\right) \rightarrow H^{3}(Q, \mathrm{Z}) \rightarrow H^{3}(Q, \mathrm{Z}) \ldots
$$

The second arrow here is a Bockstein homomorphism which we call $\beta^{\prime}$. Exactness implies that $y$ is in the kernel of $\beta^{\prime}$ if and only if it satisfies (36). Thus the necessary and sufficient condition for being able to lift Adj to a $U(n)$ bundle is

$$
\beta^{\prime}(y)=0
$$

We now claim that $\beta^{\prime}(y)=[H]$. Indeed, it easily follows from our definitions that

$$
\zeta_{i j k}=y_{i j k} q_{i j} q_{j k} q_{k i}
$$

Taking the logarithm of both sides of (40) yields

$$
\log \zeta_{i j k}=\log y_{i j k}+\log q_{i j}+\log q_{j k}+\log q_{k i}+\sqrt{-1} p_{i j k},
$$

where $p_{i j k}$ are Z-valued numbers. Next we apply the Cech coboundary operator to both sides and get

$$
\begin{aligned}
& -\log \zeta_{i j k}+\log \zeta_{j k l}-\log \zeta_{i k l}+\log \zeta_{i j l} \\
& \quad=-\log y_{i j k}+\log y_{j k l}-\log y_{i k l}+\log y_{i j l}+\ldots,
\end{aligned}
$$

where the dots denote a Čech 3-coboundary with values in Z. The left-hand side is a Čech cocycle representing $\beta(\zeta) \equiv[H]$, while the right-hand side is a Čech cocycle representing $\beta^{\prime}(y)$ plus a coboundary. Thus we get

$$
\beta^{\prime}(y)=[H]
$$

An important consequence of this relation is $n[H]=0$, i.e. $[H]$ is an $n$-torsion class. Indeed, the third arrow in (38) is multiplication by $n$, so exactness implies $n \beta^{\prime} \equiv 0$, hence $n[H]=0$. 
Let us summarize what we have learned so far. The postulated transformation law for the "connection 1-forms" $\tilde{A}_{i}(24)$ leads to a welldefined vector bundle Adj of rank $n^{2}$ with structure group $S U(n) / \mathbb{Z}_{n}$. The 1-forms $A_{i}=\tilde{A}_{i}-\mu_{i}$ taken in the adjoint representation of $U(n)$ define a good connection on Adj. In general Adj carries a nontrivial t'Hooft magnetic flux $y \in H^{2}\left(Q, \mathbb{Z}_{n}\right)$ which prevents lifting it to an $S U(n)$ bundle. Even if $y \neq 0$, it may still be possible to lift Adj to a $U(n)$ bundle; the obstruction to doing this is $\beta^{\prime}(y) \in H^{3}(Q, \mathrm{Z})$. We showed that the Bockstein of $y$ is precisely $[H]$. This means that Adj can be lifted to a $U(n)$ bundle if and only if $[H]$ is trivial. (Caution: remember that all objects here are restricted to $Q$, i.e. $[H]$ is really $[H]_{Q}$.)

\section{The definition of the trace of holonomy}

In the previous section we remarked that the 1-forms $A_{i}$ enable one to compute the holonomy and its trace in the adjoint representation of $U(n)$. What we really need, however, is the trace of holonomy in the fundamental representation.

We have seen that when $\beta(\zeta) \equiv[H]$ is nonzero, it is impossible to lift the $S U(n) / \mathbb{Z}_{n}$ bundle Adj to a $U(n)$ bundle. In such a situation the 1-forms $A_{i}$ cannot be interpreted as connection 1-forms for a $U(n)$ vector bundle. Nevertheless, they do have a geometric meaning: they define a connection on an Azumaya module $\Gamma$. Roughly speaking, a connection on an Azumaya module $\Gamma$ is a connection on the underlying vector bundle which is compatible with the action of $A$ on $\Gamma$. A more precise definition goes as follows. Suppose we are given a connection on an Azumaya algebra A. This means that we are given a covariant derivative $\nabla^{\mathrm{A}}$ on the underlying vector bundle such that for any two elements $R_{1}, R_{2} \in \mathrm{A}$ and any vector field $X$ we have

$$
\nabla_{X}^{\mathrm{A}}\left(R_{1} R_{2}\right)=\nabla_{X}^{\mathrm{A}}\left(R_{1}\right) R_{2}+R_{1} \nabla_{X}^{\mathrm{A}}\left(R_{2}\right) .
$$

Then a connection on a module $\Gamma$ over $\mathrm{A}$ is a covariant derivative $\nabla^{\Gamma}$ on the underlying vector bundle such that for any $s \in \Gamma, R \in \mathrm{A}$ and any vector field $X$ we have

$$
\nabla_{X}^{\Gamma}(R s)=\nabla_{X}^{\mathrm{A}}(R) s+R \nabla_{X}^{\Gamma}(s)
$$


In terms of an open cover $\left\{\mathcal{U}_{i}\right\}$ and transition functions (2), a connection on $A$ is given by

$$
\nabla_{\mu}^{\mathrm{A}}(R)_{i}=\partial_{\mu} R_{i}-\sqrt{-1}\left[b_{i \mu}, R_{i}\right],
$$

where $b_{i}=b_{i \mu} d x^{\mu}$ are $u(m)$-valued 1-forms which satisfy on double overlaps

$$
b_{i}=G_{i j} b_{j} G_{i j}^{-1}+\sqrt{-1} G_{i j} d G_{i j}^{-1} .
$$

Then a connection on a module $\Gamma$ is given by

$$
\nabla_{\mu}^{\Gamma}(s)_{i}=\partial_{\mu} s_{i}-\sqrt{-1}\left(1 \otimes b_{i \mu}-A_{i \mu}^{T} \otimes 1\right) s_{i},
$$

where $A_{i}$ are the $u(n)$-valued 1-forms defined in the previous section. The property (45) is easily verified.

A crucial difference between a connection on the module $\Gamma$ and a connection on an ordinary vector bundle arises when one tries to define the holonomy of a connection along a loop $\gamma \in L Q$. From (29) one readily sees that any natural definition of holonomy will produce an object which is well-defined only modulo multiplication by a complex number with unit modulus. For this reason we anticipate that the trace of holonomy of $A$ will actually take values in a line bundle over $L Q$.

To define the trace of holonomy of $A$ we will work locally on $L Q$. We have already picked an open cover $\mathcal{U}_{i}, i \in I$, of $Q$. An open cover of $L Q$ will consist of sets $V_{p}, p \in \mathcal{P}$. $\mathcal{P}$ is the set of pairs $(t, f)$, where $t$ is a triangulation of $S^{1}$ and $f$ is a map from the simplices of $t$ to $I$. By definition $V_{(t, f)} \subset L Q$ is the set of loops $\gamma: S^{1} \rightarrow Q$ such that for any simplex $\sigma \in t \gamma(\sigma) \subset \mathcal{U}_{f(\sigma)}$. Now pick any $p=(t, f) \in \mathcal{P}$. Let $t$ consist of $r$ simplices $\sigma_{1}, \ldots, \sigma_{r}$. Since $S^{1}$ is oriented, the simplices are also oriented. Let $v_{\ell}, \ell=1, \ldots, r$ be the vertices of $t$ numbered so that the boundary of $\sigma_{\ell}$ is $v_{\ell+1}-v_{\ell}$. We will use a shorthand $f_{\ell}=f\left(\sigma_{\ell}\right)$.

Suppose we are given a $\gamma \in V_{p}$. We denote by $A_{\ell}$ the pull-back of the 1 -form $A_{f_{\ell}}$ to $\sigma_{\ell}$. We denote by $h_{\ell, \ell-1}$ the matrix $h_{f_{\ell} f_{\ell-1}}\left(\gamma\left(v_{\ell}\right)\right)$. We now define a function $\mathcal{F}_{p}: V_{p} \rightarrow \mathbb{C}$ by the following formula:

$$
\mathcal{F}_{p}(\gamma)=\operatorname{Tr}\left[\prod_{\ell=n}^{1} \operatorname{Hol}_{\sigma_{\ell}}\left(A_{\ell}\right) h_{\ell, \ell-1}\right]
$$

where $\operatorname{Tr}$ is the trace in the fundamental representation. 
Suppose the intersection $V_{p} \cap V_{p^{\prime}}=V_{p p^{\prime}}$ is nonempty. We want to compare $\mathcal{F}_{p}$ and $\mathcal{F}_{p^{\prime}}$ on the double overlap $V_{p p^{\prime}}$. Let $\gamma \in V_{p p^{\prime}}$; then a short computation shows that

$$
\mathcal{F}_{p}(\gamma)=\mathcal{H}_{p p^{\prime}} \mathcal{F}_{p^{\prime}}(\gamma)
$$

where $\mathcal{H}_{p p^{\prime}}$ is a $U(1)$-valued 1-cochain on $L Q$. In order to write it down we need to introduce some more notation. Let $\sigma_{1}^{\prime}, \ldots, \sigma_{r^{\prime}}^{\prime}$ be the simplices of $t^{\prime}$. We define a nondecreasing sequence of integers $i_{0}, \ldots, i_{r}$ by

$$
v_{1} \in \sigma_{i_{1}}^{\prime}, \quad v_{2} \in \sigma_{i_{2}}^{\prime}, \quad \ldots, \quad v_{r} \in \sigma_{i_{r}}^{\prime}
$$

The cochain $\mathcal{H}$ is given by

$$
\begin{aligned}
& \left(\prod_{j_{r}=i_{r}}^{j_{r}=i_{r-1}+1} \zeta_{f_{r}^{\prime} f_{r} f_{j_{r}-1}^{\prime}}\right) \zeta_{f_{i_{r-1}}^{\prime} f_{r} f_{r-1}}\left(\prod_{j_{r-1}=i_{r-1}}^{j_{r-1}=i_{r-2}+1} \zeta_{f_{j_{r-1}}^{\prime} f_{r-1} f_{j_{r-1}-1}^{\prime}}\right) \ldots \\
& \left(\prod_{j_{1}=i_{2}}^{j_{r}=i_{1}+1} \zeta_{f_{j_{1}}^{\prime} f_{1} f_{j_{1}-1}^{\prime}}\right) \zeta_{f_{i_{1}}^{\prime} f_{1} f_{r} .}
\end{aligned}
$$

From (50) it follows that on triple overlaps $V_{p p^{\prime} p^{\prime \prime}}$ the transition functions $\mathcal{H}_{p p^{\prime} p^{\prime \prime}}$ satisfy

$$
\mathcal{H}_{p p^{\prime}} \mathcal{H}_{p^{\prime} p^{\prime \prime}} \mathcal{H}_{p^{\prime \prime} p}=1
$$

In other words, $\mathcal{H}$ is a 1 -cocycle over $L Q$ with values in $U(1)$.

The cocycle $\mathcal{H}$ defines a line bundle $\mathcal{L}_{A}$ over $L Q$. The first Chern class of this bundle $c_{1}\left(\mathcal{L}_{A}\right) \in H^{2}(L Q, \mathrm{Z})$ is the image of $\mathcal{H}$ under the Bockstein homomorphism $\beta^{\prime \prime}: H^{1}(L Q, U(1)) \rightarrow H^{2}(L Q, \mathrm{Z})$. Since the transition functions $\mathcal{H}_{p p^{\prime}}$ are constant, the line bundle $\mathcal{L}_{A}$ is flat and its first Chern class is pure torsion.

We define the trace of holonomy of $A$ in the fundamental representation to be the global section of the line bundle $\mathcal{L}_{A}$ which is given locally by $\mathcal{F}_{p}$. Thus the trace of holonomy of $A$ is a section of a flat line bundle over $L Q$. 


\section{Cancellation of global anomalies for bosonic strings}

In this section we establish that the path integral for an open bosonic string ending on D-branes is well-defined if the transition functions $h_{i j}$ satisfy the twisted gluing condition (29).

Recall that $\xi$ maps $\partial \Sigma$ to a submanifold $Q \subset M$ on which the Dbranes are wrapped. From now on we will reinstate subscripts $Q$ where necessary. We assume that $[H]_{Q}$ is a torsion class.

Consider the space $\operatorname{Map}(\Sigma, M)$ of maps from the worldsheet $\Sigma$ to $M$ such that $\partial \Sigma$ is mapped to a submanifold $Q \subset M$. We are dealing with oriented strings, so $\Sigma$ and $\partial \Sigma$ are oriented. It will be sufficient to consider the case when $\partial \Sigma$ has a single component, i.e. $\Sigma$ is a Riemann surface with a single hole. If we choose a diffeomorphism $S^{1} \rightarrow \partial \Sigma$, there is a natural projection $\pi: \operatorname{Map}(\Sigma, M) \rightarrow L Q$. In the previous section we showed that the trace of holonomy of $A$ is a section of a certain line bundle $\mathcal{L}_{A}$ over $L Q$. This bundle is flat, but in general nontrivial. For string theory applications we need to consider the pullback of $\mathcal{L}_{A}$ to $\operatorname{Map}(\Sigma, M)$ by $\pi$. It turns out that the pull-back is trivial, and its trivialization is given by $\exp \left(-\sqrt{-1} \int \xi^{*} B\right)$. To prove this, we should first accurately define $\exp \left(-\sqrt{-1} \int \xi^{*} B\right)$. This has been done for worldsheets without boundaries in [13] and extended to worldsheets with boundaries in [14]. Below we describe the construction of $[13,14]$ as it applies to our particular situation. A version of this construction which does not depend on the choice of open cover is explained in Chapter 6 of [15].

We warn the reader that the accurate definition of $\exp \left(-\sqrt{-1} \int \xi^{*} B\right)$ is rather lengthy, so on first reading he or she may want to jump to the next section. The upshot is that $\exp \left(-\sqrt{-1} \int \xi^{*} B\right)$ is a trivialization of the line bundle $\pi^{*}\left(\mathcal{L}_{A}\right)$. Moreover, our definition of $\exp \left(-\sqrt{-1} \int \xi^{*} B\right)$ is such that the product of the last two factors in (13) is a well-defined function on $\operatorname{Map}(\Sigma, M)$. 


\subsection{The case of a closed worldsheet}

We will start by defining $\exp \left(-\sqrt{-1} \int \xi^{*} B\right)$ when $\Sigma$ is a closed oriented surface. In this case we want $\exp \left(-\sqrt{-1} \int \xi^{*} B\right)$ to be a well-defined function on $\operatorname{Map}(\Sigma, M)$. Moreover, we expect that this function is invariant with respect to gauge transformations $B \rightarrow B+d \lambda$, where $\lambda$ is a globally defined 1-form.

Recall that we have a good cover of $M$ indexed by a set $I$. Let $\mathcal{S}$ be the set of pairs $(\tau, \phi)$ where $\tau$ is the triangulation of $\Sigma$ and $\phi$ is a map from the simplices of $\tau$ to $I$. For any $s \in \mathcal{S}$ we define a set $W_{s} \subset \operatorname{Map}(\Sigma, M)$ consisting of all the maps $\xi: \Sigma \rightarrow M$ such that for any simplex $s \in \tau \xi(s) \subset \mathcal{U}_{\phi(s)}$. The sets $W_{s}, s \in \mathcal{S}$ form an open cover of $\operatorname{Map}(\Sigma, M)$.

We will work locally on $\operatorname{Map}(\Sigma, M)$. We want to define a collection of functions $\Phi_{s}: W_{s} \rightarrow U(1)$ indexed by elements of $\mathcal{S}$ and show that they agree on double overlaps $W_{s s^{\prime}}=W_{s} \cap W_{s^{\prime}}$. To do this we have to introduce some more notation. Let us fix a triangulation $\tau$ of $\Sigma$. Let $\left\{s_{a}\right\}, a \in \mathcal{A}$, be the set of all simplices of $\tau$, let $\left\{e_{b}\right\}, b \in \mathcal{B}$, be the set of all edges, and let $\left\{v_{c}\right\}, c \in \mathcal{C}$ be the set of all vertices. Any edge belongs to exactly two simplices. A vertex can belong to two or more simplices. We will say that $v$ is $n$-valent if it belongs to exactly $n$ simplices.

An orientation of $\Sigma$ induces an orientation on all the simplices of $\tau$. An edge $e_{a a^{\prime}}=s_{a} \cap s_{a^{\prime}}$ can be oriented if we specify an ordering of $a, a^{\prime}$.

Let us fix a map $\phi: \mathcal{A} \rightarrow I$. Let $\xi \in W_{(\tau, \phi)}$. Then we can use $\xi$ to pull back a 2 -form $B_{\phi}(a)$ on $\mathcal{U}_{\phi(a)}$ to a 2 -form $B_{a}$ on the simplex $s_{a}$. Similarly, we can pull back a 1 -form $\Lambda_{\phi(a) \phi\left(a^{\prime}\right)}$ to a 1 -form $\Lambda_{b}$ on the edge $e_{b}$, where $e_{b}$ is shared by the simplices $s_{a}$ and $s_{a^{\prime}}$. Finally, suppose we have a vertex $v$ shared by simplices $s_{a_{1}}, \ldots, s_{a_{n}}$. For any three indices $a, a^{\prime}, a^{\prime \prime} \in\left\{a_{1}, \ldots, a_{n}\right\}$ we will define a complex number $\alpha_{a a^{\prime} a^{\prime \prime}}$ as $\xi^{*} \alpha_{\phi(a) \phi\left(a^{\prime}\right) \phi\left(a^{\prime \prime}\right)}(v)$.

Given $s=(\tau, \phi)$ and $\xi \in W_{s}$ we now define a $U(1)$-valued function on $W_{s}$ :

$$
\tilde{\Phi}_{s}(\xi)=\exp \left[-\sqrt{-1} \sum_{a \in \mathcal{A}} \int_{s_{a}} B_{a}-\sqrt{-1} \sum_{b \in \mathcal{B}} \int_{e_{b}} \Lambda_{b}\right]
$$


The functions $\tilde{\Phi}_{s}$ are not quite what we need because they do not agree on double overlaps. However, this can be fixed by introducing a correction factor $C_{v}$ for each vertex of the triangulation which depends on the 2-cocycle $\alpha$ from (15). The correction factor is defined as follows.

1. If a vertex $v$ is divalent, $C_{v}=1$.

2. Suppose $v$ is trivalent. Since $\Sigma$ is oriented, the simplices sharing $v$ have a natural cyclic order $s_{a}, s_{a^{\prime}}, s_{a^{\prime \prime}}$. We set $C_{v}=\alpha_{a a^{\prime} a^{\prime \prime}}$.

3. Suppose the vertex $v$ belongs to $n$ simplices $s_{a_{1}}, \ldots, s_{a_{n}}, n>3$. We pick an arbitrary index $a \in\left\{a_{1}, \ldots, a_{n}\right\}$ and set

$$
C_{v}=\alpha_{a_{1} a_{2} a} \alpha_{a_{2} a_{3} a} \ldots \alpha_{a_{n} a_{1} a} .
$$

It is easy to check that $C_{v}$ does not depend on the choice of $a$ because of (16).

A patient reader should be able to prove that the functions $\Phi_{s}$ thus defined agree on double overlaps $W_{s s^{\prime}}$. The proof uses the relations (14)-(16). We define $\exp \left(-\sqrt{-1} \int \xi^{*} B\right)$ to be the global function which is given locally by $\Phi_{s}$. It is obvious that the global function thus defined is invariant with respect to gauge transformations $B \rightarrow B+d \lambda$.

\subsection{The case of a worldsheet with a boundary}

Let $\Sigma$ be a Riemann surface with a single hole such that the boundary $\partial \Sigma$ is mapped to $Q$. For any triangulation $\tau$ we divide the edges and vertices into internal and boundary ones. The sets $\mathcal{B}$ and $\mathcal{B}^{\prime}$ will label the internal and boundary edges, respectively, while the sets $\mathcal{C}$ and $\mathcal{C}^{\prime}$ will label the internal and boundary vertices, respectively. The set $\mathcal{A}$ will label the simplices of $\tau$, as before. A boundary edge belongs to exactly one simplex and has a natural orientation induced by that of $\partial \Sigma$. A boundary vertex belongs to one or more simplices. Suppose that a boundary vertex $v$ belongs to $n$ simplices $s_{a_{1}}, \ldots, s_{a_{n}}$. Orientation of $\partial \Sigma$ induces a natural order on $a_{1}, \ldots, a_{n}$ (not just cyclic order!)

We define functions $\Phi_{s}: W_{s} \rightarrow U(1)$ as a product of "internal" and "boundary" contributions. The internal contribution is the same as for a closed $\Sigma$. To define the boundary contribution we need to introduce some more notation. We use $\xi$ to pull back 1-forms $\mu_{i}$ on $\mathcal{U}_{i}$ 
to 1 -forms $\mu_{b}, b \in \mathcal{B}^{\prime}$ on $e_{b}$. Similarly, to every boundary vertex shared by the simplices $s_{a_{1}}, \ldots, s_{a_{n}}$ we associate a set of numbers $\rho_{a a^{\prime}}$ where $a, a^{\prime} \in\left\{a_{1}, \ldots, a_{n}\right\}$ by pulling back 0 -forms $\rho_{i j}$ (see (20). The boundary contribution is a product of a factor associated with boundary edges and a factor associated with boundary vertices. The first factor is

$$
\exp \left[\sqrt{-1} \sum_{b \in \mathcal{B}^{\prime}} \int_{e_{b}} \mu_{b}\right] .
$$

The second factor is a product over all boundary vertices; a factor $C_{v}$ associated with an individual vertex is defined by the following rules:

1. If the vertex $v$ is univalent, $C_{v}=1$.

2. If the vertex $v$ is divalent and belongs to an ordered pair of simplices $s_{a}$ and $s_{a^{\prime}}$, then $C_{v}=\exp \left(\sqrt{-1} \rho_{a a^{\prime}}\right)$.

3. Suppose the vertex $v$ is $n$-valent, $n>2$. Let $s_{a_{1}}, \ldots, s_{a_{n}}$ be the ordered simplices sharing $v$. We set

$$
C_{v}=\alpha_{a_{3} a_{2} a_{1}} \alpha_{a_{4} a_{3} a_{1}} \ldots \alpha_{a_{n} a_{n-1} a_{1}} \exp \left(\sqrt{-1} \rho_{a_{1} a_{n}}\right) .
$$

The functions $\Phi_{s}: W_{s} \rightarrow U(1)$ are now completely defined. It remains to compute the transition functions on double overlaps. A very patient reader should be able to prove that

$$
\Phi_{s}=\mathcal{H}_{\omega(s) \omega\left(s^{\prime}\right)} \Phi_{s^{\prime}}
$$

where $\mathcal{H}$ is the 1-cocycle (51) and $\omega$ is a natural projection $\omega: \mathcal{S} \rightarrow \mathcal{P}$ induced by the natural projection $\pi: \operatorname{Map}(\Sigma, M) \rightarrow L Q$. Recall that $\mathcal{H}$ defined a line bundle $\mathcal{L}_{A}$ over $L Q$. It follows that the collection of functions $\left\{\Phi_{s}\right\}, s \in \mathcal{S}$ define a section of the pull-back line bundle $\pi^{*}\left(\mathcal{L}_{A}\right)$.

We now define $\exp \left(-\sqrt{-1} \int \xi^{*} B\right)$ as a global section of $\pi^{*}\left(\mathcal{L}_{A}\right)$ which is given locally by $\Phi_{s}$. Since $\Phi_{s}$ take values in $U(1)$, $\exp \left(\sqrt{-1} \int \xi^{*} B\right)$ is a trivialization of $\pi^{*}\left(\mathcal{L}_{A}^{-1}\right)$. From (57) and (50) it follows that

$$
\exp \left(\sqrt{-1} \int \xi^{*} B\right) \operatorname{Tr} \operatorname{Hol}_{\partial \Sigma}\left(\xi^{*} A\right)
$$


is a well-defined function on $\operatorname{Map}(\Sigma, M)$. Hence the open string path integral is well-defined. Moreover, it is easy to see that this function is invariant with respect to gauge transformations $B \rightarrow B+d \lambda, A \rightarrow$ $A-\lambda$, where $\lambda$ is a globally defined 1-form.

The above argument can be trivially extended to the case when $\partial \Sigma$ has several components. The details are left as an exercise for the reader.

\section{Generalizations}

We are of course much more interested in superstrings rather than bosonic strings. In superstring theory $M$ is a spin manifold, while $Q \subset M$ is not necessarily spin. As explained in [1], the superstring path integral contains an extra problematic factor $\operatorname{Pfaff}(D)$, the pfaffian of the Dirac operator on the worldsheet. This object is a section of a line bundle over the space

$$
X=\operatorname{Met}(\Sigma) \times \operatorname{Map}(\Sigma, M)
$$

where $\operatorname{Met}(\Sigma)$ is the space of metrics on $\Sigma$. We will call this line bundle Pfaff. Pfaff is flat, but in general nontrivial.

One of the results of [1] is the computation of the Chern class of Pfaff. Namely, the first Chern class is given by

$$
c_{1}(\mathrm{Pfaff})=\pi^{* *} \mathcal{T}\left(W_{3}(N)\right) .
$$

Here $\pi^{\prime}$ is natural projection $\pi^{\prime}: X \rightarrow L Q, \mathcal{T}$ is the transgression homomorphism ${ }^{4} \mathcal{T}: H^{3}(Q, \mathrm{Z}) \rightarrow H^{2}(L Q, \mathrm{Z})$, and $W_{3}(N)$ is the Bockstein of the second Stiefel-Whitney class $w_{2}(N)$ of the normal bundle of $Q$. Since $W_{3}(N)$ is a 2-torsion class, $c_{1}$ (Pfaff) is also a 2-torsion class.

In Section III we have also explained that the trace of holonomy of a connection on an Azumaya module takes values in a line bundle $\mathcal{L}_{A}$

\footnotetext{
${ }^{4}$ Let us remind the definition of the transgression homomorphism. Let $x$ be a class in $H^{3}(Q, \mathrm{Z})$. Consider the evaluation map $e: S^{1} \times L Q \rightarrow Q$ given by $e:(t, \gamma) \mapsto \gamma(t)$. We can use $e$ to pull back $x$ to $S^{1} \times L Q$. The transgression of $x$ is a class in $H^{2}(L Q, Z)$ by obtained by "integrating $e^{*}(x)$ over $S^{1}$." More formally, given a homology class $a \in H_{2}(L Q, Z)$ we define $\mathcal{T}(x)(a)=e^{*}(x)(w \times a)$, where $w \in H_{1}\left(S^{1}, \mathbb{Z}\right)$ is the fundamental homology class of $S^{1}$.
} 
over $L Q$. We have explicitly computed its transition functions in terms of a 2-cocycle $\zeta$ on $Q$. Since the transition functions $\mathcal{H}_{p p^{\prime}}$ are constant, $\mathcal{L}_{A}$ is flat, but in general nontrivial. A natural question is whether one can express the Chern class of $\mathcal{L}_{A}$ directly in terms of data on $Q$. The answer to this question can be inferred from the results of $[14,15]$ where it is shown how to construct a line bundle $\mathcal{L}_{H}$ over $L Q$ given a class $[H] \in H^{3}(Q, \mathrm{Z})$. The 1-cocycle (i.e. the transition functions) defining $\mathcal{L}_{H}$ is given in Eq. (6-19) of [15]. It is easy to see that when $[H]$ is a torsion class, this cocycle is cohomologous to our cocycle $\mathcal{H}$ and therefore $\mathcal{L}_{H}$ is isomorphic to $\mathcal{L}_{A}$. It is explained in $[14,15]$ that the first Chern class of $\mathcal{L}_{H}$ (and therefore $\mathcal{L}_{A}$ ) is the transgression of $[H]$.

Consider now the product of $\operatorname{Pfaff}(D)$ and the trace of holonomy of $A$ in the fundamental representation. The first factor is a section of the line bundle Pfaff over $X$. The second factor is a section of a line bundle $\pi^{\prime *}\left(\mathcal{L}_{A}\right)$ over $X$. The Chern class of $\pi^{\prime *}\left(\mathcal{L}_{A}\right)$ is $\pi^{\prime *} \mathcal{T}(\beta(\zeta))$, where $\zeta$ is the 2-cocycle entering the twisted gluing condition (29). In Section IV we have shown that if $\beta(\zeta) \in H^{3}(Q, \mathrm{Z})$ is a restriction of a class in $H^{3}(M, \mathrm{Z})$, then $\pi^{\prime *} \mathcal{L}_{A}$ is trivial, and therefore $\pi^{* *} \mathcal{T}(\beta(\zeta))=0$. In this section we do not assume that $\beta(\zeta) \in H^{3}(Q, Z)$ is a restriction of a class in $H^{3}(M, \mathrm{Z})$, hence $\pi^{* *} \mathcal{T}(\beta(\zeta))$ need not vanish. The product of $\operatorname{Pfaff}(D)$ and the trace of holonomy of $A$ takes values in a line bundle Pfaff $\otimes \pi^{\prime *}\left(\mathcal{L}_{A}\right)$ with first Chern class

$$
\pi^{\prime *} \mathcal{T}\left(W_{3}(N)+\beta(\zeta)\right)
$$

Cancellation of global worldsheet anomalies requires this class to vanish. From Section IV we know that if

$$
W_{3}(N)+\beta(\zeta)=[H]_{Q}
$$

for some class $[H]=H^{3}(M, \mathrm{Z})$, then Pfaff $\otimes \pi^{\prime *}\left(\mathcal{L}_{A}\right)$ is trivial and its trivialization is given by $\exp \left(-\sqrt{-1} \int \xi^{*} B\right)$. Thus $(61)$ is a sufficient for cancelling the global worldsheet anomalies. It is very plausible that that it is also a necessary condition. From Section III we know that $\beta(\zeta)=$ $\beta^{\prime}(y)$, where $y \in H^{2}\left(Q, \mathbb{Z}_{n}\right)$ is the t'Hooft magnetic flux, therefore Eq. (61) is equivalent to Eq. (12).

In the special case of D9-branes the condition (12) reduces to $\beta^{\prime}(y)=$ $[H]$. This means that if $[H] \neq 0$, the bundle on D9-branes is an 
$S U(n) / \mathbb{Z}_{n}$ bundle which cannot be lifted to a $U(n)$ bundle, and the obstruction to the lift is precisely $[H]$. As explained in the introduction, this implies that a system of equal number of D9-branes and anti-D9branes defines a class in $K_{H}(M)$. Arguments analogous to those in [4] then suggest that stable D-branes in IIB string theory are classified by $K_{H}(M)$.

It is possible to extend the discussion to Type I strings. Type I strings are unoriented, so the definition of $\exp \left(-\sqrt{-1} \int \xi^{*} B\right)$ given in Section IV must be modified. In addition, for Type I strings $H=d B$ vanishes and $[H]$ is always a 2-torsion class. We will not go into the details of the anomaly cancellation mechanism for Type I strings, but it is easy to guess what the analogue of (61) should be. Consider first the case when only D9-branes are present. They carry a $\operatorname{Spin}(32) / \mathbb{Z}_{2}$ bundle which in general cannot be lifted to a $\operatorname{Spin}(32)$ bundle. The obstruction to doing this is the "generalized second Stiefel-Whitney class" $\tilde{w}_{2} \in H^{2}\left(M, \mathbb{Z}_{2}\right)[17]$. However, in order to define the open string path integral, we need the trace of holonomy in the vector representation of $\operatorname{Spin}(32)$. We expect that this object is a section of a line bundle over $L Q$ whose first Chern class is the transgression of $\beta^{\prime}\left(\tilde{w}_{2}\right)$. We thus expect that the path integral is well-defined if

$$
\beta^{\prime}\left(\tilde{w}_{2}\right)=[H]
$$

This relation was first noticed in [12].

Consider now $n$ Type I D5-branes wrapped on a submanifold $Q \subset$ $M$. It is usually said that Type I D5-branes carry a principal bundle with structure group $S p(n)$. We expect that this statement will be modified when $[H]_{Q} \neq 0$ and the bundle will be an $S p(n) / \mathbb{Z}_{2}$ bundle without $S p(n)$ structure. The obstruction to having $S p(n)$ structure is measured by a class $y \in H^{2}\left(Q, \mathbb{Z}_{2}\right)$, and we expect that cancellation of anomalies requires $W_{3}(N)+\beta^{\prime}(y)=[H]_{Q}$.

\section{Discussion}

In this paper we have shown that when the restriction of $[H]$ to the D9-brane worldvolume is nonzero, cancellation of worldsheet anomalies forces the D9-brane bundle to be an $S U(n) / \mathbb{Z}_{n}$ bundle without $U(n)$ 
structure. As a consequence of this the geometric meaning of the gauge field changes: it becomes a connection on a module over an Azumaya algebra. This fact is responsible for the appearance of twisted K-groups $K_{H}$ in string theory.

More generally, we showed that cancellation of global worldsheet anomalies imposes a correlation between the t'Hooft magnetic flux on D-branes and $[H]_{Q}$. This correlation is summarized in (11) and (12).

A natural question is whether it is possible to extend the present discussion to the case when $[H]$ is not a torsion class. Witten has argued [4] that the answer must be negative. We give two more arguments supporting this conclusion.

First, it must be clear from our discussion that when $[H]$ is not a torsion class, it is impossible to wrap any finite number of D9-branes on $M$. This means that there is no good starting point for the Sen-Witten construction of D-branes.

Second, even assuming that one can somehow make sense of an infinite number of D9-branes (and anti-D9-branes), there seems to be no good candidate for a $\mathrm{K}$-theory for general $[H]$. Indeed, for any $[H]$ one would like to find an algebra bundle over $M$ such that its K-theory classifies the D-brane charges. The structure group of this algebra bundle should be some suitable version of $S U(n) / \mathbb{Z}_{n}$ as $n \rightarrow \infty$. A natural class of algebra bundles is suggested by a theorem of Dixmier and Douady $[18,15]$ which states that algebra bundles whose fiber is the algebra of compact (or Hilbert-Schmidt, or trace class) operators on a separable infinite-dimensional Hilbert space $V$ are in one-to-one correspondence with elements of $H^{3}(M, \mathrm{Z})$. The corresponding "gauge group" is $P U(V)$, the projective unitary group of $V$. Unfortunately, the corresponding $\mathrm{K}$-theories are not the right ones. For example, the K-group corresponding to the trivial class in $H^{3}(M, \mathrm{Z})$ would be the Grothendieck group of Hilbert bundles on $M$ with structure group $U(V)$, the unitary group of $V$. But this K-group is very different from the expected answer $\tilde{K}(M)$ : it is trivial for any manifold $M$ because all Hilbert bundles over $M$ are isomorphic to a trivial one. This happens because the "gauge group" $U(V)$ is contractible [19]. More generally, one can consider bundles or sheaves of von Neumann algebras; however none of them seems to lead to an acceptable K-theory, because their automorphism groups have the wrong homotopy type. In or- 
der to get the correct K-theory, one has to work with much smaller "gauge groups." For example, one could try a subgroup of the unitary group $U^{c}(V) \subset U(V)$ whose elements have the form $1+K$, where $K$ is compact (or Hilbert-Schmidt, or trace-class.) One can show that the K-group of Hilbert bundles with structure group $U^{c}(V)$ is the same as $\tilde{K}(M) \quad[20,21]$. Unfortunately, unlike $U(n), U^{c}(V)$ does not have a center, so this K-theory does not have a twisted version.

\section{Acknowledgements}

I would like to thank Mikhail Khovanov for useful discussions. This work was supported by a DOE grant DE-FG02-90ER40542.

\section{Note}

After this paper was posted on the Los Alamos preprint archive, the relevance of the Dixmier-Douady theory for the classification of D-brane charges has been re-examined by P. Bouwknegt and W. Mathai [22]. These authors showed that the K-theory of the Dixmier-Douady algebras reduces to the ordinary $\mathrm{K}$-theory when $[H]$ is a torsion class. This result invalidates the argument made above against the relevance of Dixmier-Douady algebras to D-branes and strongly suggests that their K-theory classifies D-brane charges for general $[H]$.

\section{References}

[1] D.S. Freed and E. Witten, Anomalies in string theory with Dbranes, hep-th/9907189.

[2] M.R. Douglas and C. Hull, D-branes and the noncommutative torus, JHEP, 02 (1998), 008, hep-th/9711165;

V. Schomerus, D-branes and deformation quantization, JHEP, 06 (1999), 030, hep-th/9903205;

for a more extensive list of references see N. Seiberg and E. Witten, String theory and noncommutative geometry, hep-th/9908142. 
[3] R. Minasian and G. Moore, K-theory and Ramond-Ramond charge, JHEP, 11 (1997), 002, hep-th/9710230.

[4] E. Witten, D-branes and $K$ theory, JHEP, 12 (1998), 019, hep-th/9810188.

[5] A. Sen, $S O(32)$ spinors of type $I$ and other solitons on braneantibrane pair, JHEP, 09 (1998), 023, hep-th/9808141.

[6] J.P. Serre, Modules projectifs et espaces fibrés à fibre vectorielle, Exp., 23, Séminaire Dubreil-Pisot, Paris, 1958.

[7] R.G. Swan, Vector bundles and projective modules, Trans. Amer. Math. Soc., 105 (1962), 264-277.

[8] A. Connes, M.R. Douglas, and A. Schwarz, Noncommutative geometry and matrix theory: Compactification on tori, JHEP, 02 (1998), 003, hep-th/9711162.

[9] A. Grothendieck, Le Groupe de Brauer I, Séminaire Bourbaki, Exp., 290 (1964/1965), 01-21.

[10] P. Donovan and M. Karoubi, Graded Brauer groups and K-theory with local coefficients, IHES Pub., 38 (1970), 5.

[11] B. Farb and R.K. Dennis, Noncommutative algebra, Graduate Texts in Mathematics, 144, Springer-Verlag, New York, 1993.

[12] A. Sen and S. Sethi, The Mirror transform of type I vacua in sixdimensions, Nucl. Phys., B499 (1997), 45, hep-th/9703157.

[13] O. Alvarez, Topological Quantization And Cohomology, Commun. Math. Phys., 100 (1985), 279.

[14] K. Gawedzki, Topological Actions In Two-Dimensional Quantum Field Theories, in 'Nonperturbative Quantum Field Theories', eds. G. t'Hooft, A. Jaffe, G. Mack, P.K. Mitter, and R. Stora, NATO Series, 185, Plenum Press, (1988), 101-141.

[15] J.-L. Brylinski, Loop spaces, Characteristic Classes, and Geometric Quantization, Progress in Mathematics, 107, Birkhäuser, Boston, 1993. 
[16] N. Hitchin, Lectures on special Lagrangian submanifolds, math.dg/9907034.

[17] M. Berkooz, R.G. Leigh, J. Polchinski, J.H. Schwarz, N. Seiberg, and E. Witten, Anomalies, dualities, and topology of $D=6$ $N=1$ superstring vacua, Nucl. Phys., B475 (1996), 115, hep-th/9605184.

[18] J. Dixmier and A. Douady, Champs continues d'espaces hilbertiens et de $C^{*}$-algèbres, Bull. Soc. Math. Fr., 91 (1963), 227-284.

[19] N.H. Kuiper, The homotopy type of the unitary group of Hilbert space, Topology, 3 (1965), 19-30.

[20] A.S. Schwarz, On the homotopic topology of Banach spaces, Dokl. Akad. Nauk SSSR, 154 (1964), 61-63.

[21] R. Palais, On the homotopy type of certain groups of operators, Topology, 3 (1965), 271-279.

[22] P. Bouwknegt and V. Mathai, D-branes, B-fields and twisted $K$ theory, JHEP, 0003 (2000), 007; hep-th/0002023. 